\title{
Series Solutions of Partial Differential Equations
}

\section{Using the Complex Integral Method}

\author{
W. Robin \\ Engineering Mathematics Group \\ Edinburgh Napier University \\ 10 Colinton Road, EH10 5DT, UK
}

Copyright (C) 2014 W. Robin. This is an open access article distributed under the Creative Commons Attribution License, which permits unrestricted use, distribution, and reproduction in any medium, provided the original work is properly cited.

\begin{abstract}
The complex integral method for solving ordinary differential equations in series $[3,7,8]$ is extended to cover the series solution of partial differential equations also. The means of this extension is straightforward, with both 'ordinary' and 'Frobenius' multiple variable power series being dealt with. Standard examples of the application of the extended method(s) to first-order, second-order, third-order and simultaneous partial differential equations are provided throughout. Examples also include the series solution of a non-linear partial differential equation and the consideration of series solutions with negative powers.
\end{abstract}

Mathematics Subject Classification: 30B10, 30E20, 32A05, 32A30, 35A25, $35 \mathrm{C} 10,35 \mathrm{~F} 10,35 \mathrm{~F} 25,35 \mathrm{G} 10$

Keywords: Partial differential equations, several complex variables, series solutions, complex integrals

\section{Introduction}

In this paper we extend the application of the complex integral method for solving ordinary differential equations (ODE) in series $[3,7,8]$ to cover the power series solution of partial differential equations (PDE) also. The means of this extension, 
from the single independent variable to the several independent variables case, is quite straightforward, as we simply apply the procedure variable by variable to the particular assumed multiple variable series format in each case, with both the 'ordinary' and 'Frobenius' multiple variable series being dealt with. The method, as it is developed, is used to provide series solutions to first-order, second-order and third-order PDE and, in addition, we consider the solution of simultaneous PDE. As an added point of interest, we extend the method, when required, to the consideration of series solutions with negative powers.

There are, of course, certain differences when we enter the realm of PDE. For a start, it is of the nature of the complex integral method to produce particular solutions [7, 8]. Also, there is the problem of accounting for boundary or initial conditions; these must be dealt with in a manner consistent with the complex integral method itself. In addition to these general points, there is the practical difficulty that the recurrence relations determining the coefficients of the series solutions will be multi-variable and multi-term. And, then, if a series solution is possible, there is the ever-present (multi-variable) convergence problem. These points aside, the actual 'mechanics' of the solution process remains the same as in the ODE case: the PDE is effectively replaced by a system of (uncoupled) simple equations in one (discrete) variable, through a purely formal process.

The paper is organized as follows. In section 2 we develop the complex integral method for the solution in series of PDE in two independent variables about two ordinary points. This is followed, in section 3, by some standard examples of first-order PDE, including a nonlinear PDE and simultaneous PDEs in two independent variables, while, in section 4, we solve some standard examples of second-order PDE in two independent variables. In section 5 we develop the complex integral method for the solution of PDE in series with three independent variables and apply it to obtain a particular solution of Laplace's equation. The complex integral method for solution in series of PDE with two independent variables is itself extended, in section 6 , to the case of Frobenius series in two independent variables, including the case of PDE where a series solution with negative powers is required, and used in the solution of certain standard examples. The paper is rounded-off with a short conclusions and discussion section, section 7 .

\section{The Basic Formalism for Two Independent variables}

When seeking series solutions to ODE, using the complex integral method [3], we consider that the solution $f(z)$ may be expressed as an infinite series about an ordinary point, $z_{0}$, of the form [4] 


$$
f(z)=\sum_{m=0}^{\infty} a_{m}\left(z-z_{0}\right)^{m}
$$

with the coefficients $\left\{a_{m}\right\}_{m=0}^{\infty}$ given by Herrera's complex integral formula as [3]

$$
a_{m}=\frac{1}{[m]_{k} 2 \pi i} \oint_{C} \frac{f^{(k)}(z)}{\left(z-z_{0}\right)^{m-k+1}} d z
$$

with $C$ an appropriate closed contour [3] and where, following Ince [4], we write

$$
[m]_{k}=m(m-1)(m-2) \cdots(m-k+1)
$$

where $m=k, k+1, k+2, k+3, \ldots$, and $k=0,1,2,3, \ldots$.

When seeking to extend the complex integral method to PDEs, it is necessary to apply the basic complex integration process, successively, variable by variable. To see how this works, suppose that we seek a series solutions to a PDE with two variables, that is, we assume that the solution of our PDE, $f\left(z_{1}, z_{2}\right)$, may be expressed as an infinite series about two ordinary points, $z_{01}$ and $z_{02}$, of the form

$$
f\left(z_{1}, z_{2}\right)=\sum_{i=0}^{\infty} \sum_{j=0}^{\infty} a_{i, j}\left(z_{1}-z_{01}\right)^{i}\left(z_{2}-z_{02}\right)^{j}
$$

Then, with

$$
f^{(k, \ell)}\left(z_{1}, z_{2}\right)=\frac{\partial^{k+\ell} f\left(z_{1}, z_{2}\right)}{\partial z_{1}^{k} \partial z_{2}^{\ell}}
$$

and $f^{(0,0)}\left(z_{1}, z_{2}\right)=f\left(z_{1}, z_{2}\right)$, we can differentiate $(2.3)$ to get

$$
f^{(k, \ell)}\left(z_{1}, z_{2}\right)=\sum_{i=k}^{\infty} \sum_{j=\ell}^{\infty}[i]_{k}[j]_{\ell} a_{i, j}\left(z_{1}-z_{01}\right)^{i-k}\left(z_{2}-z_{02}\right)^{j-\ell}
$$

Or

$$
\begin{aligned}
f^{(k, \ell)}\left(z_{1}, z_{2}\right)= & {[m]_{k}[n]_{\ell} a_{m, n}\left(z_{1}-z_{01}\right)^{m-k}\left(z_{2}-z_{02}\right)^{n-\ell} } \\
& +\sum_{i=k \neq m}^{\infty} \sum_{j=\ell \neq n}^{\infty}[i]_{k}[j]_{\ell} a_{i, j}\left(z_{1}-z_{01}\right)^{i-k}\left(z_{2}-z_{02}\right)^{j-\ell}
\end{aligned}
$$

Finally, dividing through $(2.6 \mathrm{~b})$ by $\left(z_{1}-z_{01}\right)^{m-k+1}\left(z_{2}-z_{02}\right)^{n-\ell+1}$ and integrating round appropriate closed contours, $C_{2}$ and $C_{1}$, with respect to $z_{2}$ and then $z_{1}$, we find that 


$$
\oint_{C_{1}} d z_{1} \frac{1}{\left(z_{1}-z_{01}\right)^{m-k+1}} \oint_{C_{2}} d z_{2} \frac{f^{(k, \ell)}\left(z_{1}, z_{2}\right)}{\left(z_{2}-z_{02}\right)^{n-\ell+1}}=(2 \pi \hat{i})^{2}[m]_{k}[n]_{\ell} a_{m, n}
$$

where (2.6) follows from the standard result [7] that, if $v$ is an integer and $\hat{i}^{2}=-1$

$$
\oint_{C}\left(z-z_{0}\right)^{v} d z=\left\{\begin{array}{cc}
2 \pi \hat{i}, \quad n=-1 \\
0, & \text { otherwise }
\end{array}\right.
$$

where $z$ is a complex variable and $z_{0}$ a fixed point within the closed contour $C$.

Looking back, we see that $m=k, k+1, k+2, k+3, \ldots, k=0,1,2,3, \ldots$. and $n=\ell, \ell+1, \ell+2, \ell+3, \ldots, \ell=0,1,2,3, \ldots$.

Apparently, if we have higher-order (than second-order) PDE, the formula (2.7) expands accordingly, in an obvious manner (see section 5 below).

With PDE, the boundary or initial conditions play an active role in the solution process and it will prove necessary, following $[2,5,6]$, to transform the boundary or initial conditions along with the PDE. To do this we assume that the boundary or initial conditions are analytic functions of the variables and use the complex integral formulas (2.2) or (2.7) or their logical extension (when necessary) to transform them along with the PDE. As mentioned, this process follows $[2,5,6]$ and becomes more transparent as the examples presented below are workedthrough.

Before we move-on to the next section, it is convenient, at this point, to recall the definition of the Kronecker delta function, which we write in our notation as $\delta_{i, j}$, with

$$
\delta_{i, j}= \begin{cases}0, & \text { if } \mathrm{i} \neq \mathrm{j} \\ 1, & \text { if } \mathrm{i}=\mathrm{j}\end{cases}
$$

The Kronecker delta function plays a not insignificant role in what follows.

\section{First-Order PDE with Two Independent Variables}

In this and the following section, we present a number of examples, including some from the literature involving the differential transform method, which acts as benchmark or comparison for the current method, involving PDE with two independent variables. We transform the original notations into the standard notation we have introduced above and assume, henceforth, that $z_{01}=z_{02}=0$.

For our first example, we consider the first-order linear PDE [2] 


$$
z_{1} f^{(1,0)}\left(z_{1}, z_{2}\right)-f^{(0,1)}\left(z_{1}, z_{2}\right)=z_{1} z_{2}
$$

subject to the conditions

$$
f^{(0,0)}\left(z_{1}, 0\right)=\sum_{i=0}^{\infty} a_{i, 0} z_{1}^{i}=0 \text { and } f^{(0,0)}\left(0, z_{2}\right)=\sum_{j=0}^{\infty} a_{0, j} z_{2}^{j}=0
$$

We now divide through (3.1) by $z_{1}^{i+1} z_{2}^{j+1}$ and integrate round appropriate closed contours, $C_{2}$ and $C_{1}$, with respect to $z_{2}$ and then $z_{1}$, to get (using (2.8))

$$
\oint_{C_{1}} d z_{1} \frac{1}{z_{1}{ }^{i}} \oint_{C_{2}} d z_{2} \frac{f^{(1,0)}\left(z_{1}, z_{2}\right)}{z_{2}{ }^{j+1}}-\oint_{C_{1}} d z_{1} \frac{1}{z_{1}{ }^{i+1}} \oint_{C_{2}} d z_{2} \frac{f^{(0,1)}\left(z_{1}, z_{2}\right)}{z_{2}{ }^{j+1}}=\delta_{i, 1} \delta_{j, 1}(2 \pi \hat{i})^{2}
$$

The next step is to compare the integrands of (3.3) with the general result (2.7), when we find that (3.3) is transformed into

$$
i a_{i, j}-(j+1) a_{i, j+1}=\delta_{i, 1} \delta_{j, 1}
$$

We now divide through the conditions (3.2) by $z_{1}^{i+1}$ or $z_{2}^{j+1}$ and integrate round an appropriate closed contours, $C_{1}$ or $C_{2}$, with respect to $z_{1}$ or $z_{2}$, to get

$$
\oint_{C_{1}} \frac{f^{(0,0)}\left(z_{1}, 0\right)}{z_{1}^{i+1}} d z_{1}=0 \text { and } \oint_{C_{2}} \frac{f^{(0,0)}\left(0, z_{2}\right)}{z_{2}^{j+1}} d z_{2}=0
$$

or, from (2.7) and (3.2)

$$
a_{i, 0}=0 \text { and } a_{0, j}=0
$$

With (3.4) and (3.6) we have effectively recovered the solution of Chen and Ho [2], and it is a straightforward matter to check that (by mathematical induction)

$$
a_{1, j}=(-1)^{j} \frac{1}{j !}, j=2,3,4, \ldots
$$

and $a_{i, j}=0$ otherwise, so that the solution of (3.1) subject to (3.2) is

$$
f\left(z_{1}, z_{2}\right)=z_{1} \sum_{j=2}^{\infty}(-1)^{j} z_{2}^{j}
$$

For our next example, we consider the first-order nonlinear PDE [5] 


$$
f^{(0,1)}\left(z_{1}, z_{2}\right)-f^{(0,0)}\left(z_{1}, z_{2}\right) f^{(1,0)}\left(z_{1}, z_{2}\right)=0
$$

subject to the conditions

$$
f^{(0,0)}\left(z_{1}, 0\right)=\sum_{i=0}^{\infty} a_{i, 0} z_{1}^{i}=z_{1}
$$

and

$$
f^{(0,0)}\left(0, z_{2}\right)=\sum_{j=0}^{\infty} a_{0, j} z_{2}^{j}=0
$$

We now divide through (3.9) by $z_{1}^{i+1} z_{2}^{j+1}$ and integrate round appropriate closed contours, $C_{2}$ and $C_{1}$, with respect to $z_{2}$ and then $z_{1}$, to get (using (2.4))

$$
\begin{aligned}
\oint_{C_{1}} d z_{1} \frac{1}{z_{1}{ }^{i+1}} \oint_{C_{2}} d z_{2} \frac{f^{(0,1)}\left(z_{1}, z_{2}\right)}{z_{2}{ }^{j+1}} & \\
& -\sum_{s=0}^{\infty} \sum_{r=0}^{\infty} a_{r, s} \oint_{C_{1}} d z_{1} \frac{1}{z_{1}{ }^{i-r+1}} \oint_{C_{2}} d z_{2} \frac{f^{(1,0)}\left(z_{1}, z_{2}\right)}{z_{2}{ }^{j-s+1}}=0
\end{aligned}
$$

Comparing the integrands of (3.11) with the general result (2.7), we find that (3.11) is transformed into

$$
(j+1) a_{i, j+1}-\sum_{s=0}^{j} \sum_{r=0}^{i} a_{r, s}(i-r+1) a_{i-r+1, j-s}=0
$$

Meanwhile, from the conditions (3.10a) we get

$$
a_{1,0}=1 \text { and } a_{i .0}=0 \text { otherwise }
$$

and condition (3.10b) is then an identity. Substituting (3.13) into (3.12), we find that

$$
(j+1) a_{1, j+1}-\sum_{s=0}^{j} a_{1, s} a_{1, j-s}=0
$$

so that, on combining (3.13) and (3.14), the solution of our difference problem (3.12) with (3.13) is

$$
a_{i, j}=\left\{\begin{array}{l}
(-1)^{j}, \text { if } i=1 \\
0, \quad \text { otherwise }
\end{array} \text { for } j=0,1,2,3, \ldots\right.
$$

Finally, from (3.15) the solution of (3.9), subject to the conditions (3.10), is 


$$
f\left(z_{1}, z_{2}\right)=z_{1} \sum_{j=0}^{\infty}(-1)^{j} z_{2}^{j}=\frac{z_{1}}{1+z_{2}}
$$

in agreement with Jang et al [5] (and (3.9) and (3.10)).

The complex integral method may be applied to systems of PDE with equal facility as its application to individual PDE. Consider the following system of first-order PDE [6]

$$
f^{(0,1)}\left(z_{1}, z_{2}\right)-g^{(1,0)}\left(z_{1}, z_{2}\right)+f^{(0,0)}\left(z_{1}, z_{2}\right)+g^{(0,0)}\left(z_{1}, z_{2}\right)=0
$$

and

$$
g^{(0,1)}\left(z_{1}, z_{2}\right)-f^{(1,0)}\left(z_{1}, z_{2}\right)+f^{(0,0)}\left(z_{1}, z_{2}\right)+g^{(0,0)}\left(z_{1}, z_{2}\right)=0
$$

where

$$
f\left(z_{1}, z_{2}\right)=\sum_{i=0}^{\infty} \sum_{j=0}^{\infty} a_{i, j} z_{1}^{i} z_{2}^{j} \text { and } g\left(z_{1}, z_{2}\right)=\sum_{i=0}^{\infty} \sum_{j=0}^{\infty} b_{i, j} z_{1}^{i} z_{2}^{j}
$$

subject to the conditions that

$$
f^{(0,0)}\left(z_{1}, 0\right)=\sinh \left(z_{1}\right)=\sum_{i=0}^{\infty} \frac{z_{1}^{2 i+1}}{(2 i+1) !}
$$

and

$$
g^{(0,0)}\left(z_{1}, 0\right)=\cosh \left(z_{1}\right)=\sum_{i=0}^{\infty} \frac{z_{1}^{2 i}}{(2 i) !}
$$

The complex integral method is applied as above. Starting with (3.17a) and (3.17b), we divide through by $z_{1}^{i+1} z_{2}^{j+1}$, integrate round appropriate closed contours, $C_{2}$ and $C_{1}$, with respect to $z_{2}$ and then $z_{1}$, and then compare the resulting integrands with the general result (2.7), when we find that equations (3.17a) and (3.17b) are transformed into

$$
(j+1) a_{i, j+1}-(i+1) b_{i+1, j}+a_{i, j}+b_{i, j}=0
$$

and

$$
(j+1) b_{i, j+1}-(i+1) a_{i+1, j}+a_{i, j}+b_{i, j}=0
$$

Next, we divide through (3.18a) and (3.18b) by $z_{1}^{i+1}$, integrate round an appropriate closed contour, $C_{1}$, with respect to $z_{1}$, and then compare the resulting 
integrand with the general result (2.7), when we find that equations (3.18a) and (3.18b) are transformed into

$$
a_{i, 0}=\frac{1}{(2 i+1) !}, \quad \mathrm{i}=0,1,2,3, \ldots
$$

and

$$
b_{i, 0}=\frac{1}{(2 i) !}, \quad \mathrm{i}=0,1,2,3, \ldots
$$

Equations (3.19) and (3.20) correspond to equations (7) to (10) of Ravi Kanth and Aruna [6], so that the solutions of equations (3.17a) and (3.17b), subject to the conditions (3.18a) and (3.18b), are [6]

$$
f\left(z_{1}, z_{2}\right)=\sinh \left(z_{1}-z_{2}\right)
$$

and

$$
g\left(z_{1}, z_{2}\right)=\cosh \left(z_{1}-z_{2}\right)
$$

\section{Second-Order PDE with Two Independent Variables}

In the theory of PDE the equation

$$
f^{(1,1)}\left(z_{1}, z_{2}\right)-f^{(0,0)}\left(z_{1}, z_{2}\right)=0
$$

subject to the condition that

$$
f^{(0,0)}(0,0)=1
$$

plays a role analogous to the defining equation for the exponential function in the theory of ordinary differential equations (ODE) [1]. As our first problem in this section, we seek a solution of (4.1) about the origin, subject to (4.2), using the complex integral method.

As before, the first step is to divide through (4.1) by $z_{1}^{i+1} z_{2}^{j+1}$ and integrate round appropriate closed contours, $C_{2}$ and $C_{1}$, with respect to $z_{2}$ and then $z_{1}$, to get

$$
\oint_{C_{1}} d z_{1} \frac{1}{z_{1}{ }^{i+1}} \oint_{C_{2}} d z_{2} \frac{f^{(1,1)}\left(z_{1}, z_{2}\right)}{z_{2}{ }^{j+1}}-\oint_{C_{1}} d z_{1} \frac{1}{z_{1}{ }^{i+1}} \oint_{C_{2}} d z_{2} \frac{f^{(0,0)}\left(z_{1}, z_{2}\right)}{z_{2}{ }^{j+1}}=0
$$


The next step is to compare the integrands of (4.3) with the general result (2.7), when we find that (4.3) is transformed into

$$
(j+1)(i+1) a_{i+1, j+1}-a_{i, j}=0
$$

Further, from (2.4) the condition (4.2) becomes

$$
a_{0,0}=1
$$

By inspection, the solution of (4.4), subject to (4.5), is

$$
a_{i, j}=\frac{1}{i !} \frac{1}{j !}, \quad i, j=0,1,2,3, \ldots
$$

so that the solution of (4.1), subject to (4.2), is (as is well known)

$$
f\left(z_{1}, z_{2}\right)=e^{z_{1}+z_{2}}
$$

For our second problem, we solve the diffusion equation

$$
f^{(0,1)}\left(z_{1}, z_{2}\right)-f^{(1,1)}\left(z_{1}, z_{2}\right)=0
$$

subject to the condition that

$$
f^{(0,0)}\left(z_{1}, 0\right)=\sin \left(z_{1}\right)=\sum_{i=0}^{\infty} \frac{(-1)^{i} z_{1}^{2 i+1}}{(2 i+1) !}=\sum_{i=0}^{\infty} \frac{z_{1}^{i}}{i !} \sin \left(\frac{i \pi}{2}\right)
$$

Again, the complex integral method is applied (4.8) and (4.9) and we find that equations (4.8) and (4.9) are transformed into

$$
(j+1) a_{i, j+1}-(i+2)(i+1) a_{i+2, j}=0
$$

and

$$
a_{i, 0}=\frac{1}{i !} \sin \left(\frac{i \pi}{2}\right), \quad i=0,1,2,3, \ldots
$$

respectively. Now, if we solve (4.10) subject to (4.11), we find that we get the coefficients in the expansion of $f^{(0,0)}\left(z_{1}, z_{2}\right)$ as

$$
a_{i, j}=\frac{(-1)^{j}}{j !} \frac{1}{i !} \sin \left(\frac{i \pi}{2}\right), \quad i, j=0,1,2,3, \ldots
$$

so that, the solution of (4.8) subject to (4.9) is 


$$
f\left(z_{1}, z_{2}\right)=e^{-z_{2} \sin \left(z_{1}\right)}
$$

Naturally, we can obtain the solutions of the last two examples by separation of variables, a much easier proposition in these cases.

Our third example [2] involves the solution of the second-order PDE

$$
f^{(0,2)}\left(z_{1}, z_{2}\right)-c^{2} f^{(2,0)}\left(z_{1}, z_{2}\right)=0
$$

with $c$ a constant (the wave equation), subject to the conditions

$$
f^{(0,0)}\left(z_{1}, 0\right)=\sum_{i=0}^{\infty} a_{i, 0} z_{1}^{i}=z_{1}^{3} \text { and } f^{(0,1)}\left(z_{1}, 0\right)=\sum_{i=0}^{\infty} a_{i, 1} z_{1}^{i}=z_{1}
$$

As before, we divide through (4.14) by $z_{1}^{i+1} z_{2}^{j+1}$ and integrate round appropriate closed contours, $C_{2}$ and $C_{1}$, with respect to $z_{2}$ and then $z_{1}$, to get

$$
\oint_{C_{1}} d z_{1} \frac{1}{z_{1}^{i+1}} \oint_{C_{2}} d z_{2} \frac{f^{(0,2)}\left(z_{1}, z_{2}\right)}{z_{2}{ }^{j+1}}-c^{2} \oint_{C_{1}} d z_{1} \frac{1}{z_{1}^{i+1}} \oint_{C_{2}} d z_{2} \frac{f^{(2,0)}\left(z_{1}, z_{2}\right)}{z_{2}{ }^{j+1}}=0
$$

Once again, the next step is to compare the integrands of (4.16) with the general result (2.7), when we find that (4.16) is transformed into

$$
(j+2)(j+1) a_{i, j+2}-c^{2}(i+2)(i+1) a_{i, j+1}=0
$$

Next, we divide through the conditions (4.15) by $z_{1}^{i+1}$ and integrate round an appropriate closed contour, $C_{1}$, with respect to $z_{1}$ when we get

$$
\oint_{C_{1}} \frac{f^{(0,0)}\left(z_{1}, 0\right)}{z_{1}^{i+1}} d z_{1}=\oint_{C_{1}} \frac{z_{1}^{3}}{z_{1}^{i+1}} d z_{1} \text { and } \oint_{C_{1}} \frac{f^{(0,1)}\left(z_{1}, 0\right)}{z_{2}^{i+1}} d z_{2}=\oint_{C_{1}} \frac{z_{1}}{z_{1}^{i+1}} d z_{1}
$$

or, from (2.8) and (4.15)

$$
a_{i, 0}=\delta_{i, 3} \text { and } a_{i, 1}=\delta_{i, 1}
$$

From (4.17) and (4.19) and mathematical induction we find that the only nonzero coefficients are $a_{3,0}=1, a_{1,1}=1$ and $a_{1,2}=3 c^{2}$, in agreement with [2]. This means that the (non-separable) solution of (4.14) satisfying the conditions (4.15) is [2]

$$
f\left(z_{1}, z_{2}\right)=z_{1} z_{2}+3 c^{2} z_{1} z_{2}^{2}+z_{1}^{3}
$$


For our last example in this section, following [2] again, we consider the second-order PDE

$$
f^{(0,2)}\left(z_{1}, z_{2}\right)-f^{(2,0)}\left(z_{1}, z_{2}\right)-z_{1} f^{(0,0)}\left(z_{1}, z_{2}\right)=z_{1}
$$

subject to the conditions

$$
f^{(0,0)}\left(z_{1}, 0\right)=\sum_{i=0}^{\infty} a_{i, 0} z_{1}^{i}=0 \text { and } f^{(0,1)}\left(z_{1}, 0\right)=\sum_{i=0}^{\infty} a_{i, 1} z_{1}^{i}=0
$$

Following the previous procedure, we find that (4.21) and (4.22) transform into the difference equation

$$
(j+2)(j+1) a_{i, j+2}-(i+2)(i+1) a_{i, j+1}-a_{i-2, j}=\delta_{i, 1} \delta_{j, 0}
$$

subject to the conditions that

$$
a_{i, 0}=0 \text { and } a_{i, 1}=0
$$

It is straightforward to check that the first few nonzero coefficients are

$$
a_{1,2}=\frac{1}{2}, a_{1,6}=\frac{1}{120} \text { and } a_{3,4}=\frac{1}{24}
$$

in agreement with [2] again, so that the first few terms of the solution of the initial value problem (4.21)/(4.22) are [2]

$$
f\left(z_{1}, z_{2}\right)=\frac{z_{1} z_{2}^{2}}{2}+\frac{z_{1}^{3} z_{2}^{4}}{24}+\frac{z_{1} z_{2}^{6}}{120}+\cdots
$$

\section{Second-Order PDE with Three Independent Variables}

We assume that the solution, $f\left(z_{1}, z_{2}, z_{3}\right)$, of our PDE with three independent variables may be expressed as an infinite series about three ordinary points, $z_{01}$, $z_{02}$ and $z_{03}$, of the form

$$
f\left(z_{1}, z_{2}, z_{3}\right)=\sum_{i=0}^{\infty} \sum_{j=0 l=0}^{\infty} \sum_{i, j, l}^{\infty}\left(z_{1}-z_{01}\right)^{i}\left(z_{2}-z_{02}\right)^{j}\left(z_{3}-z_{03}\right)^{l}
$$

Then, with

$$
f^{(h, k, \ell)}\left(z_{1}, z_{2}, z_{3}\right)=\frac{\partial^{h+k+\ell} f\left(z_{1}, z_{2}, z_{3}\right)}{\partial z_{1}^{h} \partial z_{2}^{k} \partial z_{3}^{\ell}}
$$


and $f^{(0,0,0)}\left(z_{1}, z_{2}, z_{3}\right)=f\left(z_{1}, z_{2}, z_{3}\right)$, we can differentiate (5.1) to get $f^{(h, k, \ell)}\left(z_{1}, z_{2}, z_{3}\right)=\sum_{i=h}^{\infty} \sum_{j=k l=\ell}^{\infty} \sum^{\infty}[i]_{h}[j]_{k}[l]_{\ell} a_{i, j}\left(z_{1}-z_{01}\right)^{i-h}\left(z_{2}-z_{02}\right)^{j-k}\left(z_{3}-z_{03}\right)^{l-\ell}$

or

$$
\begin{aligned}
& f^{(h, k, \ell)}\left(z_{1}, z_{2}, z_{3}\right)=[m]_{h}[n]_{k}[p]_{\ell} a_{m, n, p}\left(z_{1}-z_{01}\right)^{m-h}\left(z_{2}-z_{02}\right)^{n-k}\left(z_{3}-z_{03}\right)^{p-\ell} \\
& +\sum_{i=h \neq m}^{\infty} \sum_{j=k \neq n}^{\infty} \sum_{l=\ell \neq p}^{\infty}[i]_{h}[j]_{k}[l]_{\ell} a_{i, j}\left(z_{1}-z_{01}\right)^{i-h}\left(z_{2}-z_{02}\right)^{j-k}\left(z_{3}-z_{03}\right)^{l-\ell}
\end{aligned}
$$

Finally, dividing through (5.3b) by

$$
\left(z_{1}-z_{01}\right)^{m-h+1}\left(z_{2}-z_{02}\right)^{n-k+1}\left(z_{3}-z_{03}\right)^{p-\ell+1}
$$

and integrating round appropriate closed contours, $C_{3}, C_{2}$ and $C_{1}$, with respect to $z_{3}, z_{2}$ and then $z_{1}$, we find that

$$
\begin{array}{r}
\oint_{C_{1}} \frac{1}{\left(z_{1}-z_{01}\right)^{m-h+1}} \oint_{C_{2}} d z_{1} \frac{1}{\left(z_{2}-z_{02}\right)^{n-k+1}} \oint_{C_{3}} d z_{3} \frac{f^{(h, k, \ell)}\left(z_{1}, z_{2}, z_{3}\right)}{\left(z_{3}-z_{03}\right)^{l-\ell+1}} \\
=(2 \pi \hat{i})^{3}[m]_{h}[n]_{k}[p]_{\ell} a_{m, n, p}
\end{array}
$$

where (5.4) follows from the standard result (2.8). Equation (5.4) is a straightforward generalization of equation (2.7).

We restrict ourselves to the single example of solving a PDE with three independent variables; we consider Laplace's equation

$$
f^{(2,0,0)}\left(z_{1}, z_{2}, z_{3}\right)+f^{(0,2,0)}\left(z_{1}, z_{2}, z_{3}\right)+f^{(0,0,2)}\left(z_{1}, z_{2}, z_{3}\right)=0
$$

subject to the condition

$$
f^{(0,0,0)}(0,0,0)=1
$$

As before, we now take $z_{01}=z_{02}=z_{03}=0$, and following the basic method, we divide through (5.5) by $z_{1}^{m+1} z_{2}^{n+1} z_{3}^{l+1}$, and integrate to get

$$
\oint_{C_{1}} \frac{1}{z_{1}^{m+1}} \oint_{C_{2}} d z_{1} \frac{1}{z_{2}^{n+1}} \oint_{C_{3}} d z_{3} \frac{f^{(2,0,0)}\left(z_{1}, z_{2}, z_{3}\right)}{z_{3}^{l+1}}
$$




$$
\begin{aligned}
& +\oint_{C_{1}} \frac{1}{z_{1}^{m+1}} \oint_{C_{2}} d z_{1} \frac{1}{z_{2}^{n+1}} \oint_{C_{3}} d z_{3} \frac{f^{(0,2,0)}\left(z_{1}, z_{2}, z_{3}\right)}{z_{3}^{l+1}} \\
& \quad+\oint_{C_{1}} \frac{1}{z_{1}^{m+1}} \oint_{C_{2}} d z_{1} \frac{1}{z_{2}^{n+1}} \oint_{C_{3}} d z_{3} \frac{f^{(0,0,2)}\left(z_{1}, z_{2}, z_{3}\right)}{z_{3}^{l+1}}=0
\end{aligned}
$$

which, on comparison with (5.4), reduces to

$$
(i+2)(i+1) a_{i+2, j, l}+(j+2)(j+1) a_{i, j+2, l}+(l+2)(l+1) a_{i, j, l+2}=0
$$

Now, from (5.1) we see that (5.6) becomes

$$
a_{0,0,0}=1
$$

and, by inspection, we see that the solution of (5.8), subject to (5.9), is

$$
a_{i, j, l}=\frac{1}{i !} \frac{i}{j !} \frac{1}{l !}, \quad i, j, l=0,1,2,3, \ldots
$$

and recognize, via (5.1), that the solution of (5.5), subject to (5.6), is

$$
f\left(z_{1}, z_{2}, z_{3}\right)=e^{z_{1}+z_{2}+z_{3}}
$$

As in two previous examples, the solution (5.11) of (5.5), subject to (5.6), is a variable separable solution.

\section{Solution of PDE Using Frobenius Series}

In this section we consider the extension of the complex integral method to the solution of PDE in two independent variables through (initially) the assumption that the solution of the PDE has the form of a 'double' Frobenius series, that is

$$
f\left(z_{1}, z_{2}\right)=\sum_{i=0}^{\infty} \sum_{j=0}^{\infty} a_{i, j}\left(z_{1}-z_{01}\right)^{i+r}\left(z_{2}-z_{02}\right)^{j+s}
$$

where, by an analogous argument to that of section 2 , we find that

$$
\oint_{C_{1}} d z_{1} \frac{1}{\left(z_{1}-z_{01}\right)^{m+r-k+1}} \oint_{C_{2}} d z_{2} \frac{f^{(k, \ell)}\left(z_{1}, z_{2}\right)}{\left(z_{2}-z_{02}\right)^{n+s-\ell+1}}=(2 \pi \hat{i})^{2}[m+r]_{k}[n+s]_{\ell} a_{m, n}
$$


As a first example of this approach to the solution of PDE, we consider, again equation (4.1) [1] (written slightly differently)

$$
f^{(1,1)}\left(z_{1}, z_{2}\right)=f^{(0,0)}\left(z_{1}, z_{2}\right)
$$

Applying the usual integration approach with $z_{01}=z_{02}=0$, but dividing (6.3) by $z_{1}^{i+r+1} z_{2}^{j+s+1}$ this time, we integrate through (6.3) to get

$$
\oint_{C_{1}} \frac{d z_{1}}{z_{1}^{m+r+1}} \oint_{C_{2}} d z_{2} \frac{f^{(1,1)}\left(z_{1}, z_{2}\right)}{z_{2}^{n+s+1}}=\oint_{C_{1}} \frac{d z_{1}}{z_{1}^{m+r+1}} \oint_{C_{2}} d z_{2} \frac{f^{(0,0)}\left(z_{1}, z_{2}\right)}{z_{2}^{n+s+1}}
$$

which, on comparison with (6.2), reduces to

$$
(i+r+1)(j+s+1) a_{i+1, j+1}=a_{i, j}
$$

or

$$
(i+r)(j+s) a_{i, j}=a_{i-1, j-1}
$$

Equation (6.5b) is the recurrence relation for our assumed Frobenius series solution (6.1). To get the associated indicial equation, we set $i=j=0$ in (6.5b) and, with $a_{0,0} \neq 0$, we see that the indicial equation for $(6.3)$ is

$$
r s=0
$$

so that either $r=s=0$ (covered already), or $r=0$ while $s$ is arbitrary, or $s=0$ while $r$ is arbitrary. Following [1], we may take it that the general solution will, in some way, follow as a linear superposition of all three possible solution types. As we have considered the $r=s=0$ case already, we look next at the $s=0$ while $r$ is arbitrary case. From (6.5b) with $s=0$, we get

$$
a_{i, j}=\frac{a_{i-1, j-1}}{(i+r) j}
$$

so that we get the first few coefficients, recursively, as

$$
a_{0,0}, \quad a_{1,1}=\frac{a_{0,0}}{(1+r) .1}, \quad a_{2,2}=\frac{a_{0,0}}{(1+r)(2+r) .1 .2}, \ldots
$$

At this point we are at liberty to choose particular forms for $a_{0,0}$ and $r$, so we follow Chaundy [1] and choose $r=\frac{1}{a_{0,0}}=a$, when (4.8) becomes 


$$
\frac{1}{a}, \quad a_{1,1}=\frac{1}{a(a+1) \cdot 1}, \quad a_{2,2}=\frac{1}{a(a+1)(a+2) \cdot 1 \cdot 2}, \ldots
$$

and the first few terms of a particular solution of (6.3) are then

$$
f_{a}\left(z_{1}, z_{2}\right)=\frac{z_{1}^{a}}{a}+\frac{z_{1}^{a+1} z_{2}}{a(a+1) \cdot 1}+\frac{z_{1}^{a+2} z_{2}^{2}}{a(a+1)(a+2) .1 .2}+\cdots
$$

When we consider the other option, with $r=0$ while $s$ is arbitrary, we get a (third) series which, on choosing $s=\frac{1}{a_{0,0}}=b$, can be written as

$$
f_{b}\left(z_{1}, z_{2}\right)=\frac{z_{2}^{b}}{b}+\frac{z_{1} z_{2}^{b+1}}{1 \cdot b(b+1)}+\frac{z_{1}^{2} z_{2}^{b+2}}{1.2 \cdot b(b+1)(b+2)}+\cdots
$$

It follows that linear superposition of (4.7) $(r=s=0),(6.10)$ and (6.11) will produce a more general solution of the linear PDE (6.3).

Sometimes we require a Frobenius series with decreasing powers [1], for example

$$
f\left(z_{1}, z_{2}\right)=\sum_{i=0}^{\infty} \sum_{j=0}^{\infty} a_{i, j}\left(z_{1}-z_{01}\right)^{i+r}\left(z_{2}-z_{02}\right)^{-j+s}
$$

where, by an analogous argument to that of section 2, we find that

$$
\oint_{C_{1}} d z_{1} \frac{1}{\left(z_{1}-z_{01}\right)^{m+r-k+1}} \oint_{C_{2}} d z_{2} \frac{f^{(k, \ell)}\left(z_{1}, z_{2}\right)}{\left(z_{2}-z_{02}\right)^{-n+s-\ell+1}}=(2 \pi \hat{i})^{2}[m+r]_{k}[-n+s]_{\ell} a_{m, n}
$$

As an example of the application of (6.12) and (6.13), we consider, again, the diffusion equation

$$
f^{(0,1)}\left(z_{1}, z_{2}\right)-f^{(2.0)}\left(z_{1}, z_{2}\right)=0
$$

Dividing through (6.14) by $z_{1}^{i+r+1} z_{2}^{-j+s+1}$ and integrating through as usual we find that (6.14) becomes

$$
\oint_{C_{1}} \frac{d z_{1}}{z_{1}^{i+r+1}} \oint_{C_{2}} d z_{2} \frac{f^{(0,1)}\left(z_{1}, z_{2}\right)}{z_{2}^{-j+s+1}}-\oint_{C_{1}} \frac{d z_{1}}{z_{1}^{i+r+1}} \oint_{C_{2}} d z_{2} \frac{f^{(2,0)}\left(z_{1}, z_{2}\right)}{z_{2}^{-j+s+1}}=0
$$

which equation, on comparison with (6.13), reduces to the recurrence relation 


$$
(i+r+2)(i+r+1) a_{i+2, j}=-(j-s-1) a_{i, j-1}
$$

or

$$
(i+r)(i+r-1) a_{i, j}=-(j-s-1) a_{i-2, j-1}
$$

Setting $i=j=0$ in (6.16b), we get, with $a_{0,0} \neq 0$, the indicial equation

$$
r(r-1)=0
$$

so that $r=0$ or $r=1$, with $s$ arbitrary. To get the first series solution to (6.14), we set $r=0$ in (6.16b) and, with $a_{0,0}=1$, we solve (6.16b) recursively find that the first few terms of the first solution (6.12) to (6.14) are

$$
f\left(z_{1}, z_{2}\right)=z_{2}^{s}-\frac{(-s)}{2 !} z_{1}^{2} z_{2}^{s-1}+\frac{(-s)(-s+1)}{4 !} z_{1}^{4} z_{2}^{s-2}-\frac{(-s)(-s+1)(-s+2)}{6 !} z_{1}^{6} z_{2}^{s-3} \pm \cdots
$$

with the full series being given by Chaundy [1]. Similarly, to get the second Frobenius series solution to (6.14), we set $r=1$ in (6.16b) and, with $a_{0,0}=1$, we solve (6.16b) recursively find that the first few terms of the second solution (6.12) to $(6.14)$ are

$$
f\left(z_{1}, z_{2}\right)=z_{1} z_{2}^{s}-\frac{(-s)}{3 !} z_{1}^{3} z_{2}^{s-1}+\frac{(-s)(-s+1)}{5 !} z_{1}^{5} z_{2}^{s-2}-\frac{(-s)(-s+1)(-s+2)}{7 !} z_{1}^{7} z_{2}^{s-3} \pm \cdots
$$

with (again) the full series being given by Chaundy [1], who also exposes the relationship between the two series (6.18) and (6.19).

\section{Discussion and Conclusions}

Herrera's complex integral method appears to have sufficient flexibility to enable its generalization to the production of (power) series solutions to most types of differential equation. The extension processes appear 'natural' and the overall (formal) simplicity of the 'reduction' of the original equation or equations to a system of uncoupled simple equations in one variable is maintained throughout the various types of extension. The three main problems of the method, convergence of the series, solution of the recurrence relation and the provision of particular solutions only, are endemic to the power series approach anyway, and are shared by all other power series approaches to the solution of differential equations, for example, the differential transform method $[2,5,6]$. 
Many of the examples considered here were taken from papers using the differential transform method of solution of differential equations $[2,5,6]$. While both the differential transform method and the complex integral method yield the same answers in the situations where the differential transform method applies, the complex integral method is, I feel, easier to use, the methodology springing, as it does, from the general properties of the (complex) integral, as well as the basic integral 'Herrera' results (2.2), (2.7) and so on. In addition, the complex integral method generalizes, in an obvious manner, to encompass solutions of PDE inFrobenius series and series with negative powers. Indeed, Herrera's complex integral representation of the derivative of a function [3] seems like a natural generalization of the basic idea of the differential transform concept also.

Some general remarks on the applications considered above seem appropriate at this point in the discussion. First, the conditions accompanying the PDE that we solved in sections three to six were all quite simple, with the problems all being initial value problems. As shown in Jang et al [5], who apply the differential transform method, boundary value problems are amenable to the series methods considered here as well. Secondly, the complex integral method is quite capable of handling PDE with an arbitrary number of independent variables and generalorder derivatives. The generalization of the formulae presented here is quite straightforward, as we have already pointed out. Thirdly, when tackling PDE it may often be the case that a search for similarity variables will prove fruitful. For example, in solving the diffusion equation, (6.14), in section 6 , it is useful to know of the existence of the similarity variable $z_{2} / \sqrt{z_{1}}$ or, as was actually assumed, $z_{2}^{2} / z_{1}$.

In conclusion, we have extended the complex integral method for solving ordinary differential equations in series $[3,7,8]$ to cover the series solution of partial differential equations also. We have presented examples of the application of the extended method(s) to first-order, second-order, third-order, non-linear and simultaneous partial differential equations. Examples [1] involving the Frobenius' series solution of PDE, including the consideration of series solutions with negative powers, were also presented. A brief comparison with the differential transform method $[2,5,6]$ has been given and the possibility of further applications of the complex integral method mentioned. 


\section{References}

[1] Chaundy T. W.: A Method of Solving Certain Linear Partial Differential Equations. Proc. London Math. Soc. Series 2, 21 (1923) 214-234.

[2] Chen K.C. and Ho S.H.: Solving partial differential equations by twodimensional differential transform method. App. Math. Comp. 106 (1999) 171-179.

[3] Herrera J. C.: Power series solutions in nonlinear mechanics. Brookhaven National Laboratory Report 37494. Undated.

[4] Ince E. L.: Ordinary Differential Equations. Dover, New York (1956).

[5] Jang M-J., Chen C-L. and Liu Y-C.: Two-dimensional differential transform for partial differential equations. App. Math, Comp. 121 (2001) 261-270.

[6] Ravi Kanth A.S.V. and Aruna K.: Differential transform method for solving linear and non-linear systems of partial differential equations. Phys. Lett. A 372 (2008) 6896-6898.

[7] Robin W.: Series Solution of Second-Order Linear Homogeneous Ordinary Differential Equations via Complex Integration. International Mathematical Forum 9 (2014) 967-976.

[8] Robin W.: Frobenius Series Solution of Fuchs Second-Order Ordinary Differential Equations via Complex Integration. International Mathematical Forum 9 (2014) 953-965.

\section{Received: August 7, 2014}

